\title{
Quarkonia production in the STAR experiment
}

\author{
Barbara Trzeciak ${ }^{1, a}$, on behalf of the STAR Collaboration \\ ${ }^{1}$ Faculty of Nuclear Sciences and Physical Engineering, Czech Technical University in Prague, Brehova 7, \\ 11519 Praha 1, Czech Republic
}

\begin{abstract}
In this paper, we highlight recent STAR J/ $\psi$ and $\Upsilon$ results. $\mathrm{J} / \psi$ nuclear modification factors $\left(R_{A A}\right)$ in $\mathrm{Au}+\mathrm{Au}$ collisions at $\sqrt{s_{N N}}=200,62.4$ and $39 \mathrm{GeV}$ and in $\mathrm{U}+\mathrm{U}$ collisions at $\sqrt{s_{N N}}=193 \mathrm{GeV}, \Upsilon R_{A A}$ in $d+\mathrm{Au}$ and $\mathrm{Au}+\mathrm{Au}$ collisions at $\sqrt{s_{N N}}=200$ and in $\mathrm{U}+\mathrm{U}$ collisions at $\sqrt{s_{N N}}=193 \mathrm{GeV}$ are measured and compared to different theoretical calculations. We also report $\mathrm{J} / \psi$ elliptic flow $\left(v_{2}\right)$ results in $\mathrm{Au}+\mathrm{Au}$ collisions at $\sqrt{s_{N N}}=200 \mathrm{GeV}$ and the first $\psi(2 S)$ to $\mathrm{J} / \psi$ ratio measurement in $p+p$ collisions at $\sqrt{s}=$ $500 \mathrm{GeV}$.
\end{abstract}

\section{Introduction}

Studies of production of quarkonium states in heavy-ion collisions can provide insight into the thermodynamic properties of the hot and dense medium [1]. It was proposed that due to the Debye screening of the quark-antiquark potential in the hot medium quarkonia are dissociated and thus this "melting" can be a signature of the QGP formation [2]. Since different quarkonium states have different binding energies, they are expected to dissociate at different temperatures and can be treated as a QGP thermometer [3]. But there are other mechanisms that can alter quarkonium yields in heavy-ion collisions relative to $p+p$ collisions, for example statistical recombination of heavy quark-antiquark pairs in the QGP or cold nuclear matter (CNM) effects - shadowing, final state nuclear absorption, initial-state parton energy loss. Therefore, it is difficult to isolate the color screening effect from other effects. $\Upsilon$ are considered as a cleaner probe of the hot medium, compared to $\mathrm{J} / \psi$, due to negligible co-mover absorption and statistical recombination $[4,5]$ at RHIC energies. High- $p_{T} \mathrm{~J} / \psi(>5 \mathrm{GeV} / c)$ production is also expected to be almost unaffected by the recombination and CNM effects [6].

Systematic measurements of the quarkonium production as a function of centrality and transverse momentum, for different colliding systems and collision energies may help to understand the quarkonium production mechanisms in heavy-ion collisions as well as the medium properties. In this work, we present STAR measurements of the inclusive $\mathrm{J} / \psi$ production in $\mathrm{Au}+\mathrm{Au}$ collisions at $\sqrt{s_{N N}}=200$, 62.4 and $39 \mathrm{GeV}$ and in $\mathrm{U}+\mathrm{U}$ collisions at $\sqrt{s_{N N}}=193 \mathrm{GeV} \cdot \Upsilon(1 S+2 S+3 S)$ and $\Upsilon(1 S)$ measurements have been performed in $p+p, d+\mathrm{Au}$ and $\mathrm{Au}+\mathrm{Au}$ collisions at $\sqrt{s_{N N}}=200 \mathrm{GeV}$ and in $\mathrm{U}+\mathrm{U}$ collisions at $\sqrt{s_{N N}}=193 \mathrm{GeV}$. Also, the $\mathrm{J} / \psi$ elliptic flow $\left(v_{2}\right)$ results in Au+Au collisions at $\sqrt{s_{N N}}=200 \mathrm{GeV}$ are shown. $\mathrm{J} / \psi v_{2}$ measurement allows to test the hypothetical $\mathrm{J} / \psi$ production via the recombination. We also show the first measurement of $\psi(2 S)$ to $\mathrm{J} / \psi$ ratio which helps to constrain the feed-down contribution from the excited states to the inclusive $\mathrm{J} / \psi$ production.

\footnotetext{
a e-mail: trzecbar@fjfi.cvut.cz
} 


\section{Measurements of quarkonia in STAR}

Quarkonia are measured in STAR via the di-electron decay channel. The STAR detector [7] is a multipurpose detector that has large acceptance at mid-rapidity, $|\eta|<1$ with a full azimuthal coverage. Electrons can be identified using the Time Projection Chamber (TPC) [8] through ionization energy loss $(d E / d x)$ measurement. The Time Of Flight (TOF) detector [9] greatly enhances the electron identification capability at low momenta where the $d E / d x$ bands for electrons and hadrons cross each other. At high $p_{T}$ electron identification can be improved by the Barrel Electromagnetic Calorimeter (BEMC) [10] which measures electron energy. The BEMC is also used to trigger on high- $p_{T}$ electrons (HT trigger). Minimum bias (MB) data are triggered by the Vertex Position Detectors (VPD) [11].

\section{$2.1 \mathrm{~J} / \psi$ measurements}

The left panel of Fig. 1 shows STAR J/ $\psi p_{T}$ spectra in Au+Au collisions at $\sqrt{s_{N N}}=200 \mathrm{GeV}$ for different centrality bins $[12,13]$ as solid symbols. The spectra are compared to a Tsallis Blast-Wave (TBW) prediction assuming that $\mathrm{J} / \psi$ flows like lighter hadrons $[14,15]$, shown as dashed lines. At low $p_{T}(\lesssim 2 \mathrm{GeV} / c)$ the $\mathrm{J} / \psi p_{T}$ spectra are softer than the TBW prediction, which suggests that recombination may contribute to low- $p_{T} \mathrm{~J} / \psi$ production. The shape of the spectra can be also explained if $\mathrm{J} / \psi$ has small or no radial flow since the TBW fit with radial flow $\beta=0$ (solid lines) can describe the measured spectra well.
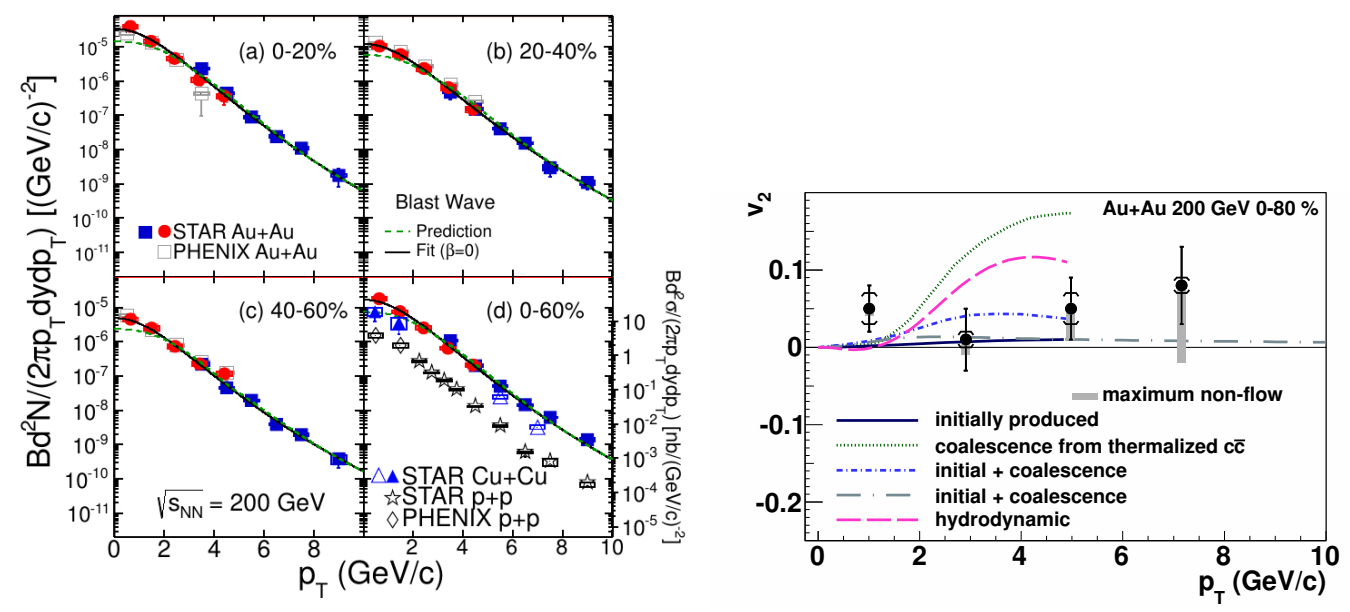

Figure 1. Left: $\mathrm{J} / \psi p_{T}$ spectra in $\mathrm{Au}+\mathrm{Au}$ collisions at $\sqrt{s_{N N}}=200 \mathrm{GeV}$ at mid-rapidity, for different centrality bins, with Tsallis Blast-Wave predictions $[14,15]$. Panel (d) shows also $p+p$ results as stars and $\mathrm{Cu}+\mathrm{Cu}$ results as triangles. Right: $\mathrm{J} / \psi v_{2}$ in $\mathrm{Au}+\mathrm{Au}$ collisions at $\sqrt{s_{N N}}=200 \mathrm{GeV}$ at mid-rapidity in $0-80 \%$ central events [16] with different model predictions ([17-20]). The gray boxes represent a non-flow estimation.

Additional information about the $\mathrm{J} / \psi$ production mechanism can by obtained by measuring the $\mathrm{J} / \psi$ elliptic flow $\left(v_{2}\right)$. The right panel of Fig. 1 shows $\mathrm{J} / \psi v_{2}$ measured in Au+Au collisions at $\sqrt{s_{N N}}$ $=200 \mathrm{GeV}$ where gray boxes represent an estimation of the maximum contribution from non-flow effects [16]. At $p_{T}>2 \mathrm{GeV} / c v_{2}$ is consistent with zero. Compared to different model predictions [1720], data disfavor the scenario that $\mathrm{J} / \psi$ with $p_{T}>2 \mathrm{GeV} / c$ are dominantly produced by recombination (coalescence) from thermalized $c \bar{c}$ pairs. 
Figure 2 shows $\mathrm{J} / \psi$ measurements as a function of the number of participant nucleons $\left(N_{\text {part }}\right)$ at various colliding energies for different colliding systems. In the left panel, results on $R_{A A}$ in $\mathrm{Au}+\mathrm{Au}$ collisions at $\sqrt{s_{N N}}=200 \mathrm{GeV}$ are presented, separately for low- $(<5 \mathrm{GeV} / c)$ [12] and high- $p_{T}(>5$ $\mathrm{GeV} / c$ ) [13] regions. We observe that the suppression increases with the collision centrality and that the $R_{A A}$ at high $p_{T}$ is systematically higher than the low- $p_{T}$ one. As it is discussed in the Introduction section, high- $p_{T} \mathrm{~J} / \psi$ are expected to be less affected by the CNM and recombination effects. The strong suppression of high- $p_{T} \mathrm{~J} / \psi$ in central collisions (0-30\%) is consistent with the expectation of the color screening effect, and thus suggests the formation of the QGP. The measured $R_{A A}$ is compared with two model predictions, Zhao and Rapp [6] and Liu et al. [21]. Both take into account direct $\mathrm{J} / \psi$ production with the color screening effect and production via recombination of $c$ and $\bar{c}$ quarks. At low $p_{T}$ both predictions (green lines) are in agreement with the data, while the high- $p_{T}$ result is well described by the Liu et al. model and the model of Zhao and Rapp underpredicts the measured $R_{A A}$.
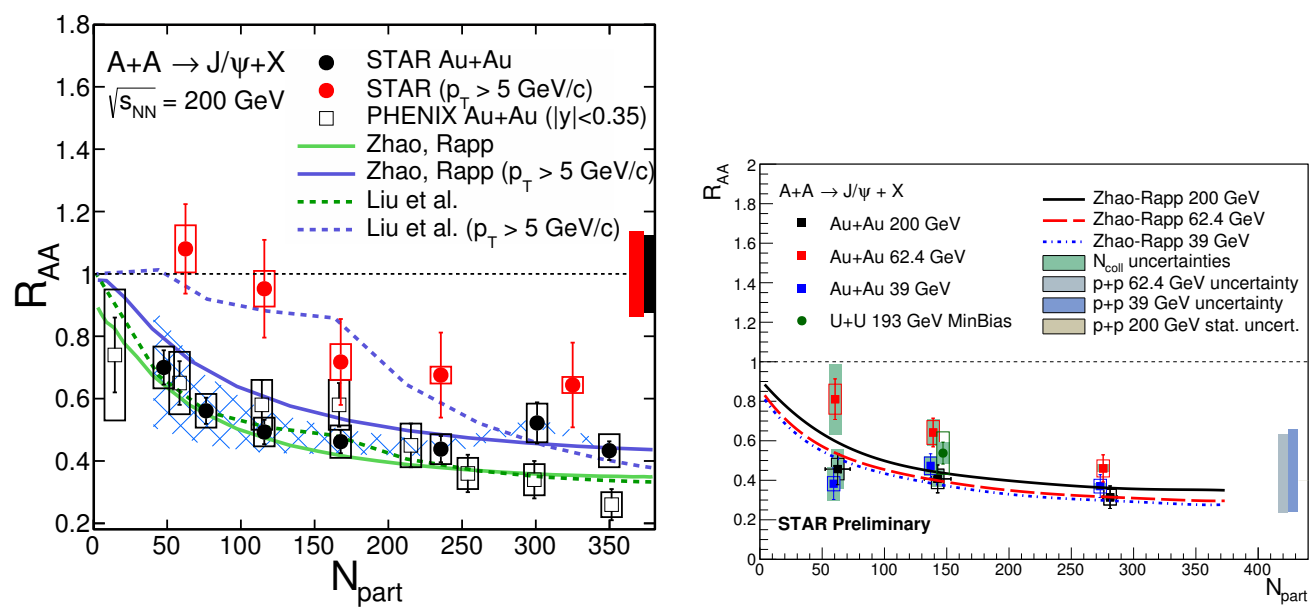

Figure 2. Left: $\mathrm{J} / \psi R_{A A}$ as a function of $N_{\text {part }}$ in $\mathrm{Au}+\mathrm{Au}$ collisions at $\sqrt{s_{N N}}=200 \mathrm{GeV}$ at mid-rapidity ([12, 13]) with two model predictions $([6,21])$. The low- $p_{T}(<5 \mathrm{GeV} / c)$ result is shown as black full circles and the high$p_{T}(>5 \mathrm{GeV} / c)$ measurement as red full circles. Right: $\mathrm{J} / \psi R_{A A}$ as a function of $N_{\text {part }}$ in $\mathrm{Au}+\mathrm{Au}$ collisions at $\sqrt{s_{N N}}=200$ (black), 62.4 (red) and 39 (blue) $\mathrm{GeV}$ at mid-rapidity with model predictions. As the green circle the minimum bias $\mathrm{U}+\mathrm{U}$ measurement at $\sqrt{s_{N N}}=193 \mathrm{GeV}$ is also presented.

When changing energies of colliding heavy ions one expects different interplay between $\operatorname{direct} \mathrm{J} / \psi$ production (with color screening), CNM and recombination effects. The right panel of Fig. 2 shows low- $p_{T} \mathrm{~J} / \psi R_{A A}$ in $\mathrm{Au}+\mathrm{Au}$ collisions for different colliding energies, $\sqrt{s_{N N}}=200$ (black), 62.4 (red) and 39 (blue) GeV. Within the uncertainties, a similar level of suppression is observed for all three energies, which can be described by the model predictions of Zhao and Rapp [6]. However, it should be noted that due to lack of precise $p+p$ measurements at 62.4 and $39 \mathrm{GeV}$ Color Evaporation Model calculations [22] are used as baselines, which introduce large uncertainties shown as boxes on the right panel of Fig. 2. The MB $R_{A A}$ measurement in $\mathrm{U}+\mathrm{U}$ collisions at $\sqrt{s_{N N}}=193 \mathrm{GeV}$ is shown as a full circle. In $\mathrm{U}+\mathrm{U}$ collisions one can reach up to $20 \%$ higher energy density compared to $\mathrm{Au}+\mathrm{Au}$ collisions in the same centrality bin [23]. Again, there is no difference in the observed suppression compared to other measurements presented in Fig. 2.

A significant fraction of inclusive $\mathrm{J} / \psi$ production comes from the feed-down of higher excited states $(\sim 40 \%), \psi(2 S)$ and $\chi_{C}$. STAR has performed the first $\psi(2 S)$ measurement at $\sqrt{s}=500 \mathrm{GeV}$ in 
$p+p$ collisions. Figure 3 shows $\psi(2 S) / J / \psi$ ratio from STAR (red full circle) compared to measurements of other experiments at different colliding energies, in $p+p$ and $p+\mathrm{A}$ collisions. The STAR data point is consistent with the observed trend, and no collision energy dependence of the $\psi(2 S)$ to $\mathrm{J} / \psi$ ratio is seen with current precision.

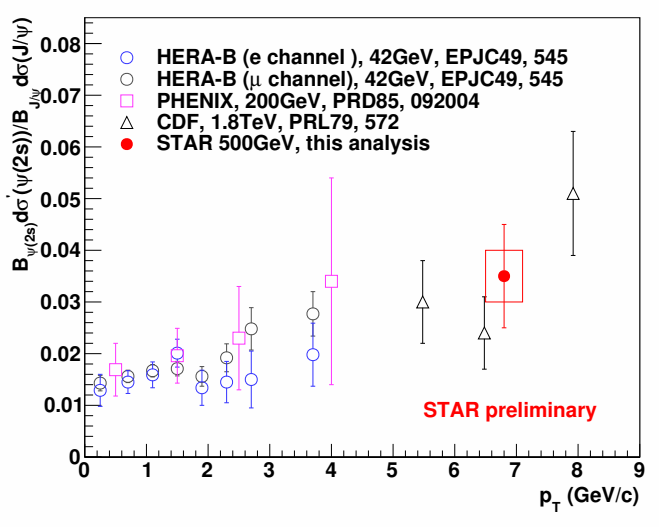

Figure 3. Ratio of $\psi(2 S)$ to $\mathrm{J} / \psi$ in $p+p$ collisions at $\sqrt{s}=500 \mathrm{GeV}$ from STAR (red circle) compared to results from other experiments at different energies.

\section{$2.2 \Upsilon$ measurements}

In order to further investigate the color screening effect, $\Upsilon$ measurements can be used. The interpretation of the observed suppression requires a $p+p$ baseline as well as knowledge of the CNM effects. Shadowing of the parton distribution functions in the nucleus or energy-loss in the nucleus can be still important in the $\Upsilon$ case and could be understood with a help of $p(d)+$ A collisions.

STAR has measured $\Upsilon$ production in $p+p, d+\mathrm{Au}$ and $\mathrm{Au}+\mathrm{Au}$ collisions at $\sqrt{s_{N N}}=200 \mathrm{GeV}$ [5] and in $\mathrm{U}+\mathrm{U}$ collisions at $\sqrt{s_{N N}}=193 \mathrm{GeV}$. The measured $p+p$ cross-section as a function of rapidity, shown in the left panel of Fig. 4, is described well by a NLO pQCD Color Evaporation Model calculation [24]. The cross-section in $\mathrm{d}+\mathrm{Au}$ collisions at forward and backward rapidity (the left panel of Fig. 4) is in agreement with the same calculation, which also takes into account the shadowing effect, but there is a discrepancy in the mid-rapidity $(y \sim 0)$ region. The nuclear modification factor $R_{d A u}$ was calculated as a function of rapidity, shown in the right panel of Fig. 4. The $R_{d A u}$ is compared to models with different assumptions regarding modification of $\Upsilon$ production in $d+$ Au collisions: Color Evaporation Model with shadowing from EPS09 parametrization for nPDF (shaded area), the model of Arleo et al. [26] where additional suppression of $\Upsilon$ is due to initial-state parton energy loss (dashed line), and the calculation that combines the energy loss and shadowing (dashed-dotted line). The STAR data point at mid-rapidity $(y \sim 0)$ is below all of these predictions which indicates additional suppression beyond the shadowing and initial-state energy loss effects.

Left panel of Fig. 5 shows $\Upsilon(1 S+2 S+3 S) R_{d A}$ and $R_{A A}$ as a function of $N_{\text {part }}$. The $d+$ Au result is presented as a green rectangle, and the $\mathrm{Au}+\mathrm{Au}$ and $\mathrm{U}+\mathrm{U}$ points for different centralities are shown as black circles and red diamonds, respectively. Au+Au and $\mathrm{U}+\mathrm{U}$ measurements follow the same trend vs $N_{\text {part }}$, with a strong suppression for the most central collisions, $R_{A u A u}=0.49 \pm 0.13(\mathrm{Au}+\mathrm{Au}$ stat. $) \pm 0.07(p+p$ stat. $) \pm 0.02$ (Au+Au syst. $) \pm 0.06(p+p$ syst. $)$ and $R_{U U}=0.35 \pm 0.17$ (stat. $)_{-0.13}^{ \pm 0.03}$ (syst.). The measured $R_{A A}$ is compared to two model predictions, Strickland and Bozow [27] and Emerick et al. [28], that include hot-nuclear-matter effects. The model of Emerick et al. takes also into account the CNM effects. Both models are in agreement with the data. 

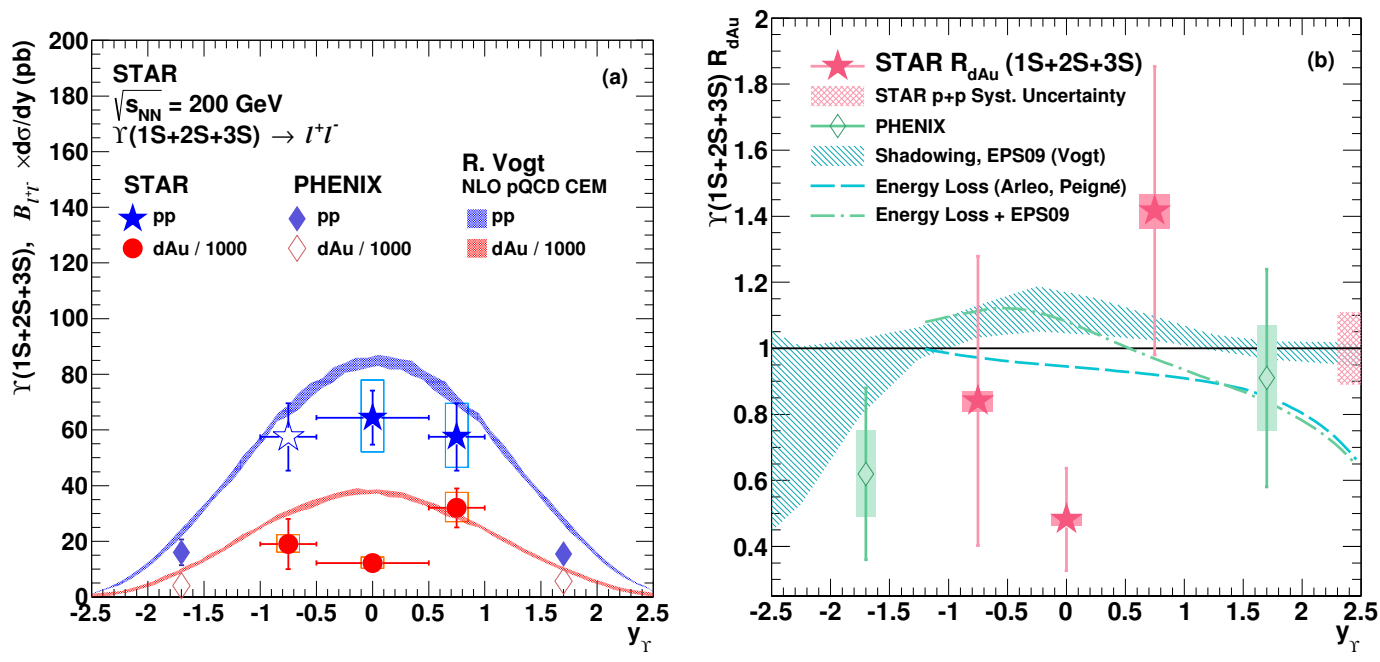

Figure 4. Left: $\Upsilon p+p$ (blue stars) and $d+\mathrm{Au}$ (red circles) cross-sections ([5]) as a function of rapidity, compared to CEM NLO pQCD calculations ([24]). Right: $R_{d A u}$ as a function of rapidity for STAR ([5]), red stars, and PHENIX ([25]), green diamond, results, compared to different model predictions ([26]).
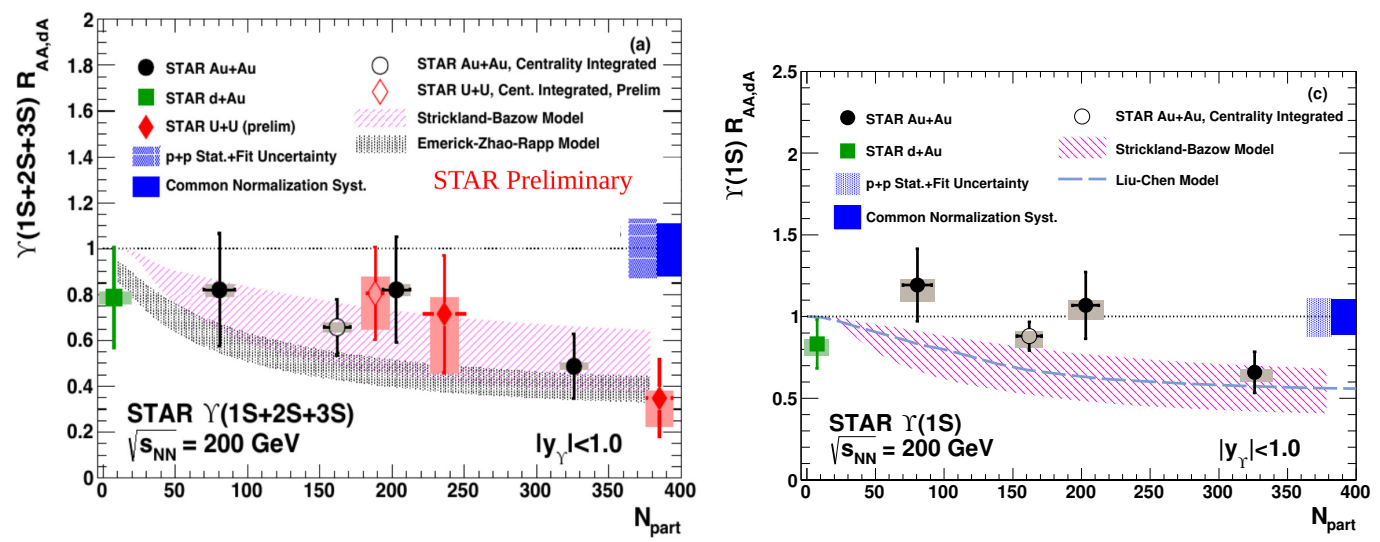

Figure 5. Left: $R_{d A}$ and $R_{A A}$ as a function of $N_{\text {part }}$ for $\Upsilon(1 S+2 S+3 S)$ at $|y|<1$ for $d+\mathrm{Au}$ (green rectangle), $\mathrm{Au}+\mathrm{Au}$ (black circles), ([5]), and $\mathrm{U}+\mathrm{U}$ (red diamonds) collisions, compared to two model predictions (shaded areas), [27, 28]. Right: $R_{d A}$ and $R_{A A}$ vs $N_{\text {part }}$ for $\Upsilon(1 S)$ ground state at $|y|<1$ for $d+\mathrm{Au}$ (green rectangle) and $\mathrm{Au}+\mathrm{Au}$ (black circles) collisions, ([5]), compared to different model predictions (shaded area and dashed line), $[27,29]$.

STAR was also able to separate the ground state $\Upsilon(1 S)$ production from the production of $\Upsilon(2 S+$ $3 S$ ). The nuclear modification factors, $R_{A A}$ and $R_{d A}$, for $\Upsilon(1 S)$ state are shown in Fig. 5, right panel, as a function of $N_{\text {part }}$. There is no suppression in $d+\mathrm{Au}$ and in peripheral and mid-central $\mathrm{Au}+\mathrm{Au}$ collisions, while for the $10 \%$ most central collisions $R_{A A}<1$. Under the assumption that $\sim 49 \%$ of $\Upsilon(1 S)$ [30] originates from the feed-down, the observed suppression in the central collisions is 
consistent with a dissociation of the higher excited states, $\Upsilon(2 S)$ and $\Upsilon(3 S)$. Also, the point at the central collisions is in good agreement with the Liu et al. model prediction [29] for inclusive $\Upsilon(1 S)$ $R_{A A}$ in which suppression is mostly due to the dissociation of the excited states. For the $\Upsilon(2 S+3 S)$ the 95\%-confidence upper limit for $R_{A A}$ in the centrality range of 0-60\% was obtained, $R_{A A}(2 S+3 S)<$ 0.32. Left panel of Fig. 6 shows the $\Upsilon(2 S+3 S)$ result on $R_{A A}$ together with $\Upsilon(1 S)$ and high- $p_{T} \mathrm{~J} / \psi$ nuclear modification factors as a function of the binding energy.
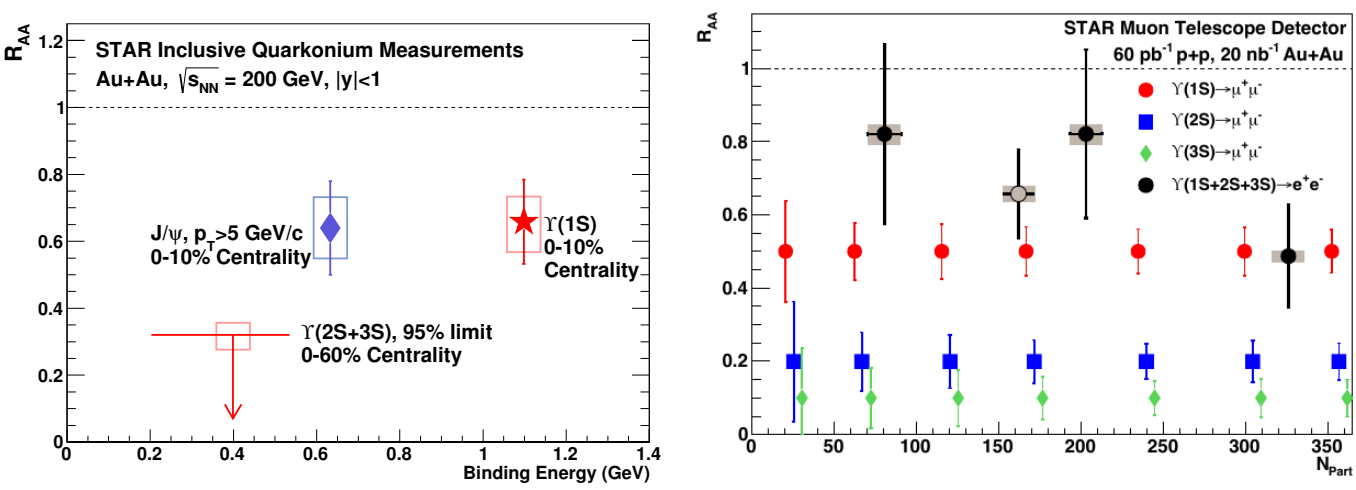

Figure 6. Left: STAR measurements of nuclear modification factors for different quarkonium states as a function of the binding energy ([5]). Right: Statistical projection of $\Upsilon R_{A A}$ measurement for different $\Upsilon$ states with the MTD detector. The figure also shows in black measured STAR $\Upsilon R_{A A}$ [5].

\section{Outlook}

STAR has two new detectors fully installed and taking data in 2014: Heavy Flavor Tracker (HFT) and Muon Telescope Detector (MTD). The HFT is a silicon vertex detector that will allow to study the non-prompt $\mathrm{J} / \psi$ production from B-meson decay, which can be identified using the displaced vertex technique. The MTD is situated outside the STAR magnet. It enables muon identification in STAR and thus quarkonium measurements in the di-muon decay channel which is cleaner than the di-electron decay channel. Compared to electrons, muons do not originate from $\gamma$ conversion in the detector material and contribution from Dalitz decays is much smaller, and they are also less affected by radiative loses in the detector material. The di-muon channel has better mass resolution that is particularly important for separation of different $\Upsilon$ states. Right panel of Fig. 6 shows a projection for $R_{A A}$ of different $\Upsilon$ states measured with the MTD.

\section{Summary}

In summary, we present recent STAR measurements on $\mathrm{J} / \psi$ and $\Upsilon$ production, as well as $\mathrm{J} / \psi v_{2}$ in $\mathrm{Au}+\mathrm{Au}$ collisions at $\sqrt{s_{N N}}=200 \mathrm{GeV}$. We have observed significant $\mathrm{J} / \psi$ production suppression in $\mathrm{Au}+\mathrm{Au}$ collisions at different colliding energies, $\sqrt{s_{N N}}=200,62.4$ and $39 \mathrm{GeV}$, and in $\mathrm{U}+\mathrm{U}$ collisions at $\sqrt{s_{N N}}=193 \mathrm{GeV}$. No strong energy dependence of the $\mathrm{J} / \psi$ suppression in $\mathrm{Au}+\mathrm{Au}$ is seen. Results on $\Upsilon$ production are also shown in $d+A u$ and $\mathrm{Au}+\mathrm{Au}$ collisions at $\sqrt{s_{N N}}=200 \mathrm{GeV}$ and in $\mathrm{U}+\mathrm{U}$ collisions at $\sqrt{s_{N N}}=193 \mathrm{GeV}$. In both $\mathrm{J} / \psi$ and $\Upsilon$ case we see similar $R_{A A}$ between $\mathrm{Au}+\mathrm{Au}$ and $\mathrm{U}+\mathrm{U}$ collisions. We observed that $\Upsilon$ and high- $p_{T} \mathrm{~J} / \psi$ are strongly suppressed in the most 
central $200 \mathrm{GeV} \mathrm{Au}+\mathrm{Au}$ collisions, which together with the indication of the complete $\Upsilon(2 S)$ and $\Upsilon(3 S)$ suppression, suggests a presence of the Quark-Gluon Plasma. Also, $\psi(2 S)$ to $\mathrm{J} / \psi$ ratio was measured for the first time in $p+p$ collisions at $\sqrt{s}=500 \mathrm{GeV}$. No collision energy dependence of the ratio is seen. New STAR upgrades will allow to perform more precise quarkonium measurements in next years.

\section{Acknowledgements}

This publication was supported by the European social fund within the framework of realizing the project ,Support of inter-sectoral mobility and quality enhancement of research teams at Czech Technical University in Prague", CZ.1.07/2.3.00/30.0034.

\section{References}

[1] A. Mocsy, P. Petreczky, Phys.Rev.Lett. 99, 211602 (2007), 0706.2183

[2] T. Matsui, H. Satz, Phys.Lett. B178, 416 (1986)

[3] A. Mocsy, Eur.Phys.J. C61, 705 (2009), 0811.0337

[4] R. Rapp, D. Blaschke, P. Crochet, Prog.Part.Nucl.Phys. 65, 209 (2010), 0807. 2470

[5] L. Adamczyk et al. (STAR Collaboration), Phys.Lett. B735, 127 (2014), 1312 . 3675

[6] X. Zhao, R. Rapp, Phys.Rev. C82, 064905 (2010), 1008. 5328

[7] K. Ackermann et al. (STAR Collaboration), Nucl. Instrum. Meth. A 499, 624 (2003)

[8] M. Anderson et al., Nucl. Instrum. Meth. A 499, 659 (2003), nucl-ex/0301015

[9] W.J. Llope et al., Nucl. Instrum. Meth. A 661, 110 (2012)

[10] M. Beddo et al. (STAR Collaboration), Nucl. Instrum. Meth. A 499, 725 (2003)

[11] W.J. Llope et al., Nucl. Instrum. Meth. A 522, 252 (2004), nucl-ex/0308022

[12] L. Adamczyk et al. (STAR Collaboration), Phys.Rev. C90, 024906 (2014), 1310. 3563

[13] L. Adamczyk et al. (STAR Collaboration), Phys. Lett. B 722, 55 (2013), 1208. 2736

[14] Z. Tang et al., Phys.Rev. C79, 051901 (2009), 0812 . 1609

[15] Z. Tang et al., Chin.Phys.Lett. 30, 031201 (2013), 1101. 1912

[16] L. Adamczyk et al. (STAR Collaboration), Phys.Rev.Lett. 111, 052301 (2013), 1212.3304

[17] L. Yan, P. Zhuang, N. Xu, Phys.Rev.Lett. 97, 232301 (2006), nucl-th/0608010

[18] V. Greco, C. Ko, R. Rapp, Phys.Lett. B595, 202 (2004), nucl-th/0312100

[19] X. Zhao, R. Rapp (2008), 0806. 1239

[20] Y. Liu, N. Xu, P. Zhuang, Nucl.Phys. A834, 317C (2010), 0910.0959

[21] Y.p. Liu, Z. Qu, N. Xu, P.f. Zhuang, Phys.Lett. B678, 72 (2009), 0901.2757

[22] R. Nelson, R. Vogt, A. Frawley, Phys.Rev. C87, 014908 (2013), 1210.4610

[23] D. Kikola, G. Odyniec, R. Vogt, Phys.Rev. C84, 054907 (2011), 1111.4693

[24] A.D. Frawley, T. Ullrich, R. Vogt, Phys.Rept. 462, 125 (2008), 0806.1013

[25] A. Adare et al. (PHENIX Collaboration), Phys.Rev. C87, 044909 (2013), 1211.4017

[26] F. Arleo, S. Peigne, JHEP 1303, 122 (2013), 1212 . 0434

[27] M. Strickland, D. Bazow, Nucl.Phys. A879, 25 (2012), 1112. 2761

[28] A. Emerick, X. Zhao, R. Rapp, Eur.Phys.J. A48, 72 (2012), 1111.6537

[29] Y. Liu, B. Chen, N. Xu, P. Zhuang, Phys.Lett. B697, 32 (2011), 1009. 2585

[30] T. Affolder et al. (CDF Collaboration), Phys.Rev.Lett. 84, 2094 (2000), hep-ex/9910025 